\title{
Panuveítis asociada a la enfermedad de Lyme en un paciente colombiano. Reporte de caso
}

\author{
Miguel Cuevas-Peláez', Alexandra Correa-García², Jorge Mario López-Mahecha²,3
}

\section{RESUMEN}

La enfermedad de Lyme es una zoonosis transmitida por la picadura de garrapatas del género Ixodes $s p$. Ha sido descrita con más frecuencia en zonas endémicas de países meriđionales. Sus manifestaciones clínicas son muy variables y se presentan de acuerdo con el estadio clínico de la enfermedad, teniendo así el compromiso ocular un espectro clínico muy variado que incluye manifestaciones desde la superficie ocular hasta la inflamación del segmento posterior. Se presenta el caso de un paciente de 72 años con un viaje reciente a los Estados Unidos (EE. UU.), atendido en la consulta particular por un oftalmólogo supraespecialista en uveítis de la ciudad de Medellín. El paciente hacía un mes refería una disminución de la agudeza visual (AV) en su ojo izquierdo y al examen oftalmológico presentó siognos clínicos de panuveítis no granulomatosa asociada a un edema macular y vasculitis retinal. Se confirmó la enfermedad de Lyme en fase tardía por la presencia de anticuerpos en sangre por Western blot. Se realizó tratamiento exitoso con antibióticos (doxiciclina), con recuperación de la AV y la mejoría completa de los siognos inflamatorios intraoculares.

\section{PALABRAS CLAVE}

Borrelia Burgdorferi; Borreliosis; Enfermedad de Lyme; Ixodes; Panuveítis; Uveítis

\section{SUMMARY}

\section{Panuveitis due to Lyme disease in a Colombian patient: Case report}

Lyme Disease is a zoonosis transmitted by arthropods that has been described with more frequency in endemic zones of meridional countries. Its clinical manifestations vary according to

Oftalmólogo general, subespecialista en inmunología ocular, Universidad de Antioquia, Medellín, Colombia.

2 Médica y cirujana. Residente del Programa de Oftalmología de la Universidad de Antioquia, Medellín, Colombia.

3 Médico y cirujano. Residente del programa de Oftalmología de la Universidad de Antioquia, Medellín, Colombia.

Correspondencia: Alexandra Correa-García; alexacoga89@gmail.com

Recibido: julio 10 de 2019

Aceptado: octubre 23 de 2019

Cómo citar: Cuevas-Peláez M, Correa-García A, López-Mahecha JM. Panuveítis asociada a la enfermedad de Lyme en un paciente colombiano. Reporte de caso. latreia. 2020 AbrJun;33(2):177-183. D0I 10.17533/udea.iatreia.46. 
the clinical stage of the disease. The eye can be affected in many forms including the involvement of the ocular surface and the posterior segment, with more severe inflammation of the retina and the choroid. We present a case of a 72-year male with a recent trip to EEUU who complained of decreasing vision in his left eye had clinical signs of non-granulomatous panureitis with macular edema and retinal vasculitis. Lyme disease was confirmed with positive antibodies in blood and Western blot test, and the patient was successfully treated using oral Doxycycline. His visual acuity improved and had a resolution of the intraocular inflammation after the specific treatment.

\section{KEY WORDS}

Borrelia Burodorferi; Borreliosis; Ixodes; Lyme Disease; Panuveitis; Uveitis

\section{INTRODUCCIÓN}

La enfermedad de Lyme es una zoonosis producida por la bacteria Borrelia burodorferi, una espiroqueta cuyo reservorio se encuentra en venados y ratones silvestres y es trasmitida a los humanos por la picadura de garrapatas del género Ixodes $s p .{ }^{(1)}$. Su distribución, tradicionalmente, ha estado circunscrita en las regiones meridionales, con una mayor prevalencia en la costa este de América del Norte y Europa central. La incidencia de la enfermedad en estas zonas puede ser mayor a 10 casos por cada 100.000 habitantes ${ }^{(2)}$.

Las manifestaciones clínicas pueden ser agudas o crónicas y se clasifican en estadios clínicos, en donde los sionos y síntomas pueden ser indolentes o no percibidos. La etapa temprana Iocalizada consta de eritema migratorio que puede estar ausente hasta en un $20 \%$ de los pacientes, sumado a síntomas sistémicos de intensidad variable como fiebre, fatiga, artralogias y mialogias ${ }^{(3)}$. La etapa temprana diseminada corresponde al periodo de la bacteriemia, donde aumentan los síntomas constitucionales y aparecen las manifestaciones neurológicas, cardiacas y oculares. En la etapa tardía predominan la artritis y encefalopatía subaguda, especialmente en los pacientes con ciertos alelos de histocompatibilidad como el HLA-DR, que expresa mayor afinidad por la proteína de superficie externa (OSpA) con aumento de la respuesta celular Th1 causando más lesión sinovial ${ }^{(4)}$.
El compromiso ocular puede ser variable y está en función de los estadios clínicos. En la etapa temprana Iocalizada predominan la conjuntivitis y el edema periorbitario. En la etapa temprana diseminada podría encontrarse queratitis, neuritis óptica con un patrón similar a las neuropatías ópticas desmielinizantes, neurorretinitis, papiledema como parte del espectro de la neuroborreliosis, neuropatías extraoculares, parálisis facial y miositis extraocular. En la etapa tardía pueden encontrarse epiescleritis, escleritis nodular, uveítis anterior, uveítis intermedia o panuveítis junto con coroiditis multifocal periférica, similar al patrón encontrado en la infección por otras espiroquetas ${ }^{(5,6)}$. El compromiso puede ser unilateral o bilateral y las manifestaciones retinocoroideas se caracterizan mejor utilizando angiografía fluoresceínica ${ }^{(7,8)}$.

La detección de anticuerpos IggG o IgM por ELISA (enzyme-linked immunosorbent assay) o por IFA (indirect immunofluorescent assay), es el método de elección para pacientes con sospecha diagnóstica que presenten síntomas sugestivos ${ }^{(9)}$. Los resultados dudosos o positivos se deben confirmar por la técnica Western blot.

No se ha establecido aún si el compromiso ocular debe tratarse como neuroborreliosis y no existen guías al respecto. La evidencia está basada en reportes de caso que han descrito el uso, principalmente, de doxiciclina, amoxicilina/clavulanato, cefixima e incluso ceftriaxona intravenosa, de acuerdo a como está indicado para el compromiso del sistema nervioso central. La duración del tratamiento es de 14 días en el compromiso ocular y hasta 28 en el manejo de la artritis por la enfermedad de Lyme.

\section{DESCRIPCIÓN DEL CASO CLÍNICO}

Paciente masculino de 72 años de edad sin antecedentes patológicos relevantes. Asistió a la consulta particular de un oftalmólogo supraespecialista en uveítis, en la ciudad de Medellín (Colombia), con cuadro de un mes de evolución de disminución indolora de la agudeza visual en su ojo izquierdo (OI), sin otros síntomas oculares asociados. Para ello recibió en otra institución altas dosis de corticosteroides por vía oral (prednisolona, 50 a 100 mg/día por 7 días) sin mejoría. También presentó artralgias de novo en ambas muñecas sin edema ni calor. Hacía 5 meses el paciente había viajado a EE. UU., para recorrer la ruta 66 de Este a Oeste. 
En el examen oftalmológico se encontró una agudeza visual mejor corregida (AVMC) en su ojo derecho (OD) de 20/30 y en su izquierdo (OI) de 20/200. Ambos ojos sin hiperemia conjuntival y con presencia de signos inflamatorios en la cámara anterior (celularidad $0,5+$, flare $1+$ ) y en la cavidad vítrea (opacidad vítrea $1+$ ) de su OI. En la prueba de fondo del ojo presentó en su OD lesiones cicatrizadas del epitelio pigmentario de la retina en la mácula y en región nasal al nervio óptico. En su OI se observó la pérdida del brillo foveal por un posible edema macular, lesiones coroideas blanquecinas de 0,7 DD en las arcadas temporales de predominio superior, algunas de ellas confluentes (Figura 1).
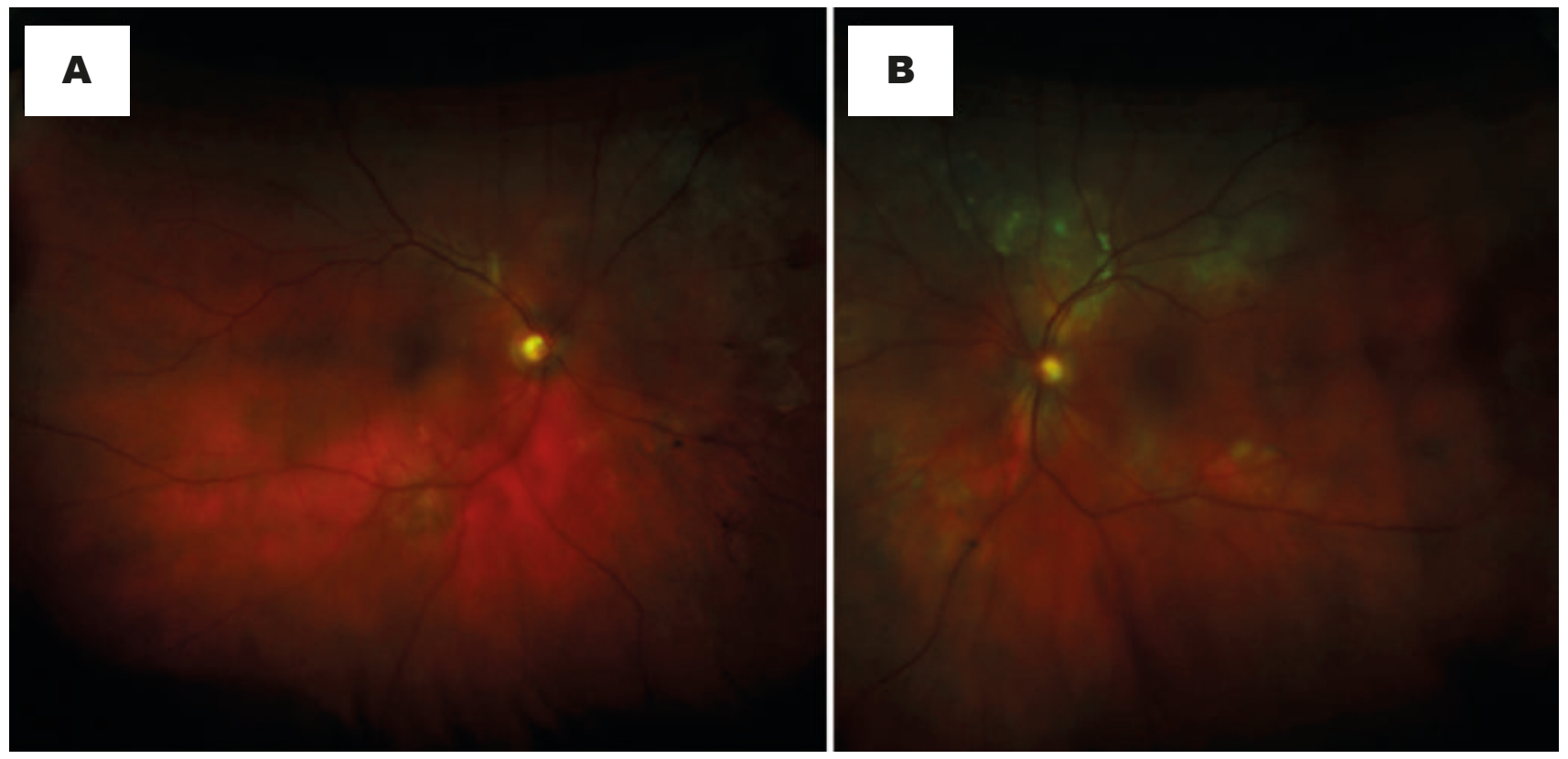

Figura 1. Fotografías de fondo de ojo a color. A. OD: mácula con cambios pigmentarios foveales escasos. Lesión hipopigmentada cicatrizada en arcada temporal inferior con pigmento. Cambios pigmentarios marcados nasales al disco. B. Ol: mácula con pérdida del brillo foveal y engrosamiento, lesiones retinocoroideas blanquecinas en las arcadas temporales, especialmente, superiores, algunas confluyentes y otras nasales y temporales al disco. Fuente: creación propia

Se realizó una angiografía con fluoresceína (AGF) que evidenció lesiones retinocoroideas cicatrizadas e inactivas en el OD e imágenes en retina y coroides sugestivas de actividad inflamatoria en su OI, con hiperfluorescencia en las fases tardías en las arcadas vasculares temporales y nasal asociadas con áreas de vasculitis retinal periférica (Fioura 2).

Los siguientes paraclínicos en la búsqueda de la enfermedad sistémica, tanto autoinmune como infecciosa, fueron negativos o estaban dentro de los límites normales: anticuerpos antinucleares, factor reumatoideo, calcio sérico, niveles de enzima convertidora de angiotensina, VDRL, FTA Abs y serologías contra el Toxoplasma gondii, herpes simple (tipo 1 y 2), Bartonella henselae, HTLV-1, citomegalovirus, Brucella y Leptospira $s p$. La radiografía de tórax no presentó ningún hallazóo patológico, también se realizó el test de Mantoux (PPD) con valor de $0 \mathrm{~mm}$. No se realizaron otras pruebas inmunológicas, porque el paciente no había mejorado con la dosis alta de prednisolona, se descartó con ello la posibilidad de tener una enfermedad autoinmune.

Debido al tipo de ureítis, a la no mejoría clínica con corticoides sistémicos y al antecedente del viaje a zonas endémicas de la enfermedad de Lyme en Estados 

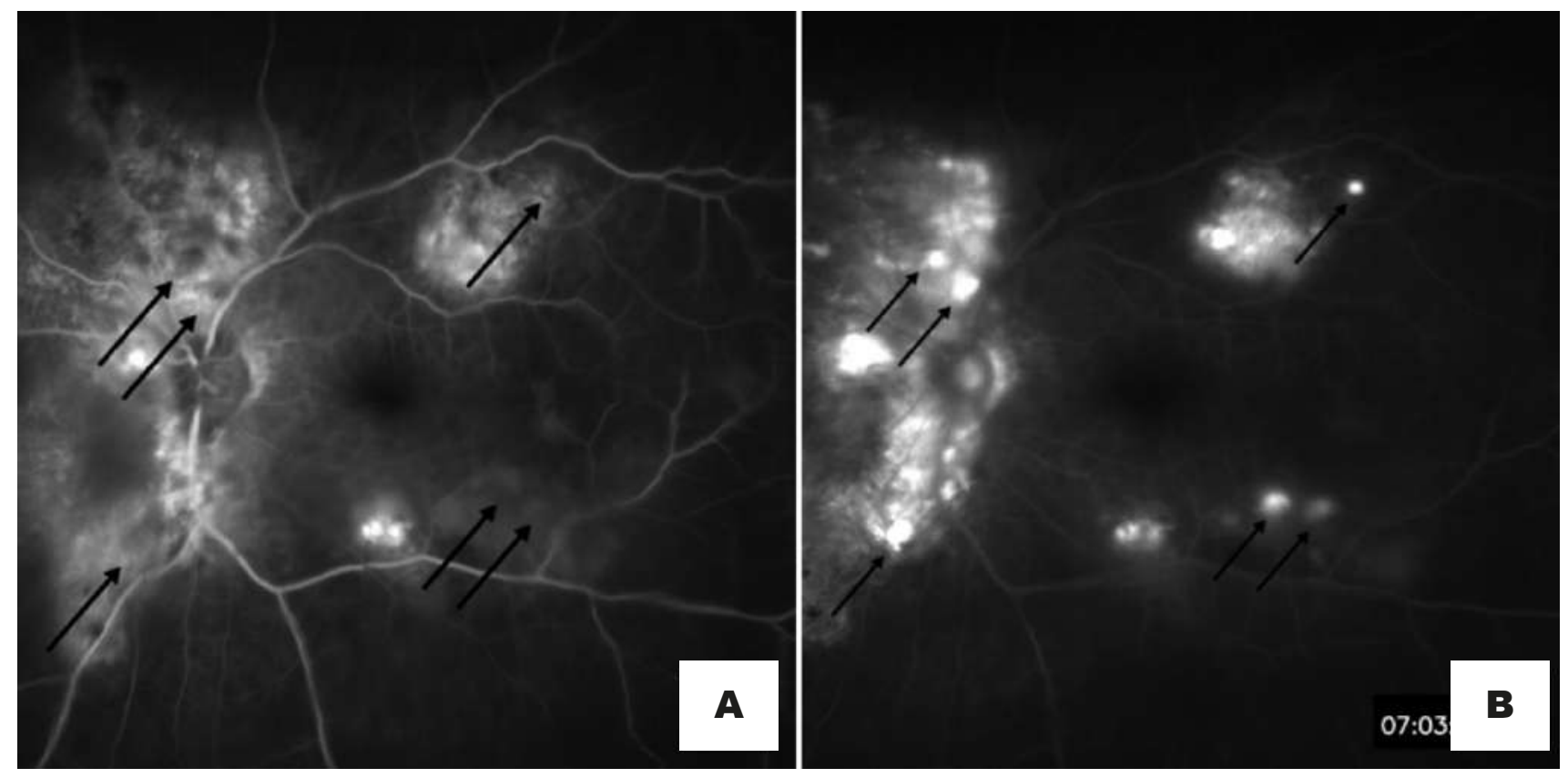

Figura 2. Angiografía con fluoresceína previa al tratamiento. A: fase de recirculación temprana, B: fase de recirculación tardía. Se observan lesiones retinocoroideas activas (flechas negras) en el polo posterior del 0l, hiperfluorescentes en fases más tardías del angiograma. Fuente: creación propia

Unidos, se solicitaron anticuerpos contra Borrelia burgdorferi. Se obtuvo IgG positivo (12,2 mg/dl) e IgM negativo. Dicho resultado fue confirmado, posteriormente, con Western blot Igg específico con valor positivo para las proteínas P22 y P31.

El paciente se trató con doxiciclina, 100 mg cada 12 horas por vía oral durante 21 días. Luego del tratamiento en controles sucesivos tuvo recuperación de la AVMC de 20/20 en el OD y 20/30 en el OI, además, de la mejoría completa de los signos inflamatorios del segmento anterior y posterior, estos últimos fueron corroborados en la AGF de control, en la cual se observaron imágenes ya cicatrizadas e inactivas en su OI con hiperfluorescencia desde las fases tempranas, además, ausencia de vasculitis retinal (Figura 3).

\section{DISCUSIÓN}

La uveítis es la inflamación del tracto ureal o capa media vascularizada y piogmentada del globo ocular.
Cuando dicha inflamación compromete el iris, el cuerpo ciliar y la coroides y/o la retina se denomina panuveítis. La principal causa de esta en Colombia es la infección por el Toxoplasma gondii, sin embargo, suele tratarse de un cuadro unilateral de aspecto granulomatoso diferente a la presentación del paciente (10). Existen otras causas de la panuveítis autoinmune o infecciosa en nuestro medio, como la sarcoidosis, el síndrome de Behcet, la enfermedad de Vogt-Koyanagi-Harada y la tuberculosis, que deben descartarse antes de pensar en diagnósticos atípicos en Colombia como la enfermedad de Lyme ${ }^{(11)}$.

En este caso, el paciente no tenía síntomas sugestivos de enfermedad sistémica, todos los estudios fueron negativos. Sin embargo, el antecedente epidemiológico y la ausencia de mejoría con la terapia antiinflamatoria sistémica aumentaban la sospecha de etiología infecciosa ${ }^{(12)}$. A excepción de las artralgias intermitentes en ambas muñecas, al parecer sin siognos de artritis, el paciente no manifestó ningún otro síntoma de borreliosis. Tampoco recordó haber 

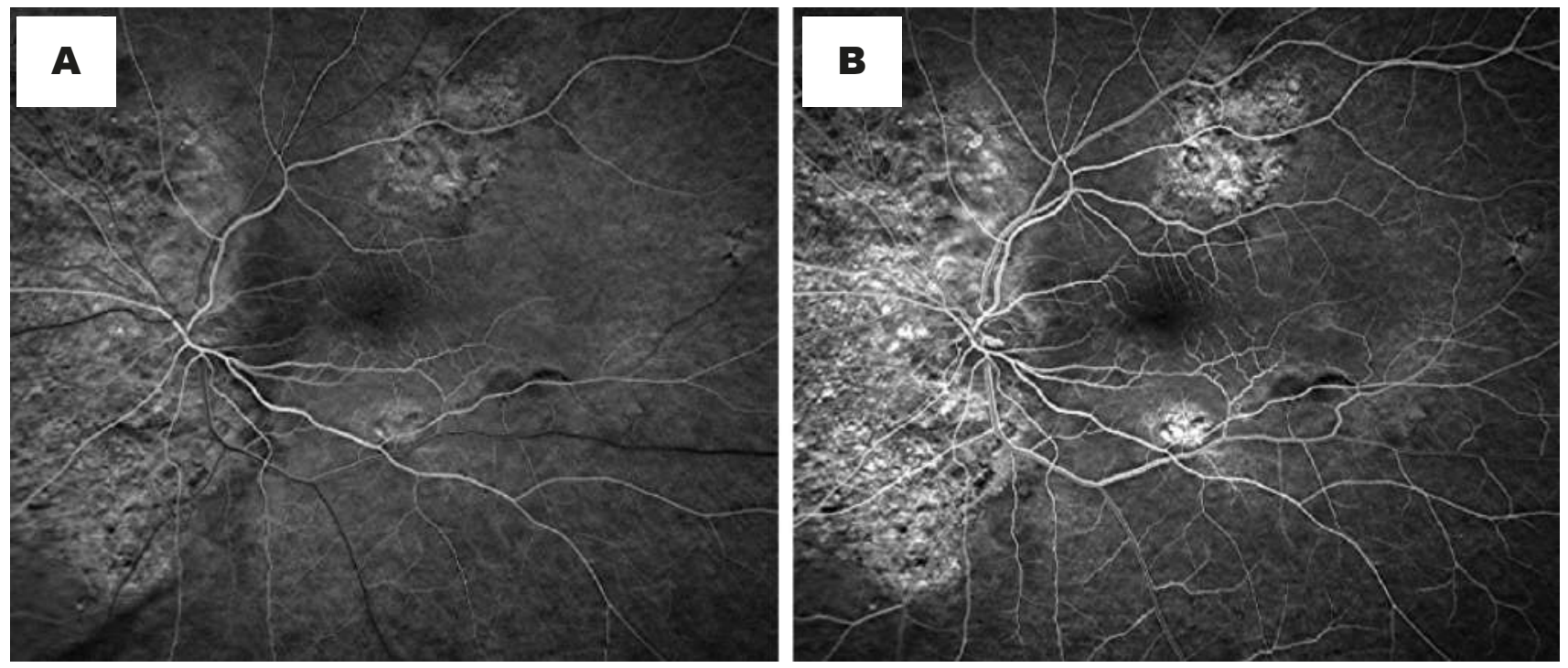

Figura 3. Angiografía con fluoresceína posterior al tratamiento. A: fase de circulación temprana, B: fase de recirculación tardía. Se observa la mejoría completa de la actividad inflamatoria de las lesiones con áreas de atrofia del epitelio pigmentario de la retina, que muestran hiperfluorescencia desde fases tempranas del angiograma del 0l. Fuente: creación propia

tenido picaduras de garrapatas o la presencia de eritema migratorio, el cual puede estar ausente o no ser recordado en el $40 \%$ de los pacientes ${ }^{(13)}$.

En cuanto a las manifestaciones oculares, en el paciente se encontró una panuveítis de tipo no granulomatoso asociada a edema macular y vasculitis retinal en su OI, con secuelas de inflamación en su OD. Estas presentaciones han sido descritas anteriormente en otros reportes de caso de la enfermedad, en fases tardías en pacientes que han sido confirmados por serología y Western blot ${ }^{(14)}$. En este caso, decidimos utilizar doxiciclina por vía oral, dada su buena tolerancia, fácil consecución y por tener una excelente evidencia en el manejo de las manifestaciones oculares de la enfermedad. Adicionalmente, se descartó el compromiso del sistema nervioso central, por ello no se consideró inđicar el uso de ceftriaxona por vía parenteral ${ }^{(15)}$.

A la fecha hay dos casos reportados en Colombia de la enfermedad de Lyme: uno en el año 2000 y otro en el 2017, ambos con antecedente de viaje a América del Norte y manifestaciones tempranas localizadas en la piel que fueron confirmados serológicamente que mejoraron con doxiciclina. Estos dos casos no presentaron manifestaciones oculares ${ }^{(16,17)}$. Por el contrario, no existen reportes de manifestaciones diseminadas o tardías de la enfermedad en Colombia, ya que, en estas etapas, las pruebas serológicas presumen el diagnóstico solo si los síntomas son sugestivos. Dada la disparidad entre pruebas comerciales y subespecies de Borrelia, algunos casos pueden pasar subdiagnosticados. Incluso existen reportes de casos con uveítis por enfermedad de Lyme en pacientes que previamente $y$ durante años fueron diagnosticados y tratados como ureítis idiopática ${ }^{(18)}$ En estos casos, los títulos serológicos decrecen con el paso del tiempo $y$, a diferencia de otras enfermedades infecciosas, no otorgan inmunidad. Por tanto, el individuo puede tener manifestaciones clínicas tempranas si se infecta de nuevo ${ }^{(19,20)}$.

A pesar de que esta zoonosis no se ha reportado como endémica en Colombia, sí se ha descrito la presencia de su vector Ixodes en la región del Carmen del Atrato y Abriaquí en Antioquia ${ }^{(21)}$, pero en ninguno de los artrópodos pudo aislarse la espiroqueta ${ }^{(22)}$. En estudios de seroprevalencia en una población del área rural y asintomática de Córdoba (Colombia), se encontraron resultados positivos para ELISA y Western blot (20 \% y $4,6 \%$, respectivamente) en ausencia de síntomas, que 
no pudieron ser explicados por reactividad cruzada con otras espiroquetas ${ }^{(23)}$.

Este es el primer caso reportado del compromiso ocuIar por la enfermedad de Lyme en Colombia. Hay que resaltar que no debe realizarse un tratamiento con corticosteroides sistémicos en pacientes con uveítis, hasta no descartarse las enfermedades de tipo infeccioso, que en este caso se sospechó por un interrogatorio exhaustivo y el tipo de compromiso intraocular. El clínico debe estar atento a este tipo de zoonosis atípica, ahora más frecuente en regiones geográficas donde no se presentaba ${ }^{(24)}$, por el movimiento migratorio y la facilidad de viajar en el mundo contemporáneo.

\section{CONFLICTOS DE INTERESES}

Ninguno por declarar. El presente artículo se ciñó a las normas vigentes para investigación médica contempladas en el marco legal colombiano en la Resolución 8430 de 1993. Contó con la aprobación deI Comité de Bioética de la Facultad de Medicina de la Universidad de Antioquia.

\section{REFERENCIAS BIBLIOGRÁFICAS}

1. Schwartz AM, Hinckley AF, Mead PS, Hook SA, Kugeler KJ. Surveillance for Lyme Disease - United States, 2008-2015. MMWR Surveill Summ. 2017;10;66(22):112. DOI 10.15585/mmwr.ss6622a1.

2. Borchers AT, Keen CL, Huntley AC, Gershwin ME. Lyme disease: a rigorous review of diagnostic criteria and treatment. J Autoimmun. 2015;57:82-115. DOI 10.1016/j.jaut.2014.09.004.

3. Hu LT. Lyme Disease. Ann Intern Med. 2016 May 3;164(9):ITC65-ITC80. DOI 10.7326/AITC201605030.

4. Bush LM, Vazquez-pertejo MT. Disease-a-Month Tick borne illness- Lyme disease. Dis Mon. 2018;64(5):195212. DOI 10.1016/j.disamonth.2018.01.007.

5. Mora P, Carta A. Ocular manifestations of Lyme borreliosis in Europe. Int J Med Sci. 2009;6(3):124-5. DOI 10.7150/ijms.6.124.

6. Mikkila H, Seppala I, Leirisalo-repo M, Immonen I, Karma A. The etiology of ureitis: The role of infections with special reference to Lyme borreliosis. Acta Ophthalmol. 1997;75(6):716-9. DOI 10.1111/j.1600-0420.1997.tb00637.x.
7. Breeveld J, Rothova A, Kuiper H. Intermediate uveitis and Lyme borreliosis. Br J Ophthalmol. 1992;76(3):1812. DOI 10.1136/bjo.76.3.181.

8. Bodaghi B. Ocular manifestations of Lyme disease. Med Mal Infect. 2007;37(7-8):518-22. DOI 10.1016/j. medmal.2006.01.016.

9. Mahne J, Kranjc BS, Strle F, Ružić-Sabljić E, Arnež M. Panuveitis caused by Borrelia burgdorferi sensu lato infection. Pediatr Infect Dis J. 2015;34(1):102-4. DOI 10.1097/INF.0000000000000473.

10. Cutler SJ, Rudenko N, Golovchenko M, Cramaro WJ, Kirpach J, Savic S, et al. Diagnosing Borreliosis. Vector Borne Zoonotic Dis. 2017;17(1):2-11. DOI 10.1089/ vbz.2016.1962.

11. Mahne J, Kranjc BS, Strle F, Ružić-Sabljić E, Arnes M. Panuveitis caused by Borrelia Burgdorferi Sensu Lato infection. Pediatr Infect Dis J. 2015 Ene;34 (1):102-4. DOI 10.1097/INF.0000000000000473.

12. De-la-Torre A1, López-Castillo CA, Rueda JC, Mantilla RD, Gómez-Marín JE, Anaya JM. Clinical patterns of uveitis in two ophthalmology centres in Bogota, Colombia. Clin Exp Ophthalmol. 2009;37(5):458-66. DOI 10.1111/j.1442-9071.2009. 02082.x.

13. Bernard Q, Jaulhac B, Boulanger N. Skin and arthropods: an effective interaction used by pathogens in vector-borne diseases. Eur J Dermatol. 2015 Apr;25 Suppl 1:18-22. DOI 10.1684/ejd.2015.2550.

14. Fajardo M, Fajardo Luis. La enfermedad de Lyme. Acta Médica Colombiana. 1994:19(4).

15. Halperin JJ. Neuroborreliosis. J Neurol. 2017 Jun;264(6):1292-97. DOI 10.1007/s00415-016-8346-2.

16. Mantilla-Flórez YF, Faccini-Martínez ÁA, Pérez-Díaz CE. American woman with early Lyme borreliosis diagnosed in a Colombian hospital. Travel Med Infect Dis. 2017;16:72-3. DOI 10.1016/j.tmaid.2017.01.005.

17. Zuluaga-de Cadena A, Boteri F, Herrera WL, Robledo J, Cortés A, Lotero MC. Enfermedad de Lyme: un caso comprobado en Colombia. Rev. CES Medicina. 2000;14(2).

18. Pleyer U, Priem S, Bergmann L, Burmester G, Hartmann C. Detection of Borrelia burgdorferi DNA in urine of patients with ocular Lyme borreliosis. Br J Ophthalmol. 2001;85(5):552-5. DOI 10.1136/bjo.85.5.552.

19. Mygland A. Could it be Lyme neuroborreliosis? Tidsskr Nor Laegeforen. 2017;137(2):86. DOI 10.4045/ tidsskr.16.1032. 
20. Pleyer U, Priem S, Bergmann L, Burmester G, Hartmann $C$. Detection of Borrelia burgdorferi DNA in urine of patients with ocular Lyme borreliosis. Br J Ophthalmol. 2001;85(5):552-5. DOI 10.1136/ bjo.85.5.552.

21. Mattar S, López Valencia G. Searching for Lyme Disease in Colombia: a Preliminary Study on the Vector. J Med Entomol. 1998;35(3):324-6.

22. Palacios R, Osorio LE, Giraldo LE, Torres AJ, Philipp MT, Ochoa MT. Positive IgG Western Blot for Borrelia burgdorferi in Colombia. Mem Inst
Oswaldo Cruz. 1999;94(4):499-503. DOI 10.1590/ S0074-02761999000400013.

23. Miranda J, Mattar S, Perdomo K, Palencia L. Seroprevalencia de Borreliosis, o Enfermedad de Lyme, en una Población Rural Expuesta de Córdoba, Colombia. Rev Salud Publica (Bogotá). 2009:11(3):480-9. DOI 10.1590/S0124-00642009000300016.

24. Stoney RJ, Esposito DH, Kozarsky P, Hamer H, Grobusch MP, Gkrania-klotsas E, et al. Infectious diseases acquired by international travellers visiting the USA. J Travel Med. 2018;25(1). DOI 10.1093/jtm/tay053. 